\title{
Article \\ Design of Powering Wireless Medical Sensor Based on Spiral-Spider Coils
}

\author{
Mustafa F. Mahmood ${ }^{1}$, Sadik Kamel Gharghan ${ }^{1, *} \mathbb{( D}$, Saleem Latteef Mohammed ${ }^{1}$, Ali Al-Naji ${ }^{1,2, *(\mathbb{D})}$ \\ and Javaan Chahl 2,3 (D) \\ 1 Electrical Engineering Technical College, Middle Technical University, Baghdad 10022, Iraq; \\ mustafa.falah@mtu.edu.iq (M.F.M.); saleem_lateef_mohammed@mtu.edu.iq (S.L.M.) \\ 2 UniSA STEM, University of South Australia, Mawson Lakes, SA 5095, Australia; Javaan.Chahl@unisa.edu.au \\ 3 Joint and Operations Analysis Division, Defence Science and Technology Group, \\ Melbourne, VIC 3207, Australia \\ * Correspondence: sadik.gharghan@mtu.edu.iq (S.K.G.); ali_al_naji@mtu.edu.iq (A.A.-N.)
}

check for updates

Citation: Mahmood, M.F.; Gharghan, S.K.; Mohammed, S.L.; Al-Naji, A.; Chahl, J. Design of Powering Wireless Medical Sensor Based on SpiralSpider Coils. Designs 2021, 5, 59. https://doi.org/10.3390/ designs5040059

Academic Editor: Sergio Saponara

Received: 3 July 2021

Accepted: 23 September 2021

Published: 26 September 2021

Publisher's Note: MDPI stays neutral with regard to jurisdictional claims in published maps and institutional affiliations.

Copyright: (c) 2021 by the authors. Licensee MDPI, Basel, Switzerland. This article is an open access article distributed under the terms and conditions of the Creative Commons Attribution (CC BY) license (https:// creativecommons.org/licenses/by/ $4.0 /)$.

\begin{abstract}
Biomedical sensors help patients monitor their health conditions and receive assistance anywhere and at any time. However, the limited battery capacity of medical devices limits their functionality. One advantageous method to tackle this limited-capacity issue is to employ the wireless power transfer (WPT) technique. In this paper, a WPT technique using a magnetic resonance coupling (MRC-WPT)-based wireless heart rate (WHR) monitoring system-which continuously records the heart rate of patients-has been designed, and its efficiency is confirmed through realtime implementation. The MRC-WPT involves three main units: the transmitter, receiver, and observing units. In this research, a new design of spiral-spider coil was designed and implemented for transmitter and receiver units, respectively, to supply the measurement unit, which includes a heart rate sensor, microcontroller, and wireless protocol (nRF24L01) with the operating voltage. The experimental results found that an adequate voltage of $5 \mathrm{~V}$ was achieved by the power component to operate the measurement unit at a $20 \mathrm{~cm}$ air gap between the receiver and transmitter coils. Further, the measurement accuracy of the WHR was $99.65 \%$ comparative to the benchmark (BM) instrument. Moreover, the measurements of the WHR were validated based on statistical analyses. The results of this study are superior to those of leading works in terms of measurement accuracy, power transfer, and Transfer efficiency.
\end{abstract}

Keywords: power transfer; spiral-spider coil; wireless heart rate sensor; wireless power transfer (WPT)

\section{Introduction}

With developments in the manufacturing of small portable medical devices, it has become necessary to create alternative energy sources to replace conventional energy sources such as fossil fuels and batteries. Alternative energy sources include wireless power transfer (WPT), piezoelectric cells, solar cells, and ultrasonic sensors that can be used in medical applications [1,2]. One of the most important energy sources is WPT. WPT can be used to transmit energy to medical devices, smart-home appliances, etc. [3,4]. Research on WPT has focused on performance in terms of efficiency and power transfer at different distances between receiver and transmitter coils. Further, its applicability to embedded medical parts and medical sensors in the field of blood-pressure monitoring, electromyography (EMG), thermometers, pacemakers (PMs), heart-rate sensors, etc., have been investigated $[5,6]$. The literature on the topic has shown that most methods for power transfer depend on the transfer distance, including far-field and near-field WPT. Near-field WPT can be used for small and mid-range distances, such as magnetic resonator coupling (MRC), capacitive coupling (CC), and inductive coupling (IC) [7-11]. Conversely, far-field WPT uses radio frequencies (RFs) to operate ultra-low-power medical sensors. 
Most research on IC-WPT uses it to enhance power transfer and efficiency at different air-gap distances between coils [12-15]. One study used MRC-WPT to improve the Transfer efficiency for small household appliances and implanted devices [16].

The coupling factor in WPT technique-based medical devices can be controlled through specific schemes, such as zero voltage switching (ZVS), as presented in [17]. In [18], the WPT system was implemented in an implanted device for pigs. The distance between the coils of the receiver and transmitter could be increased with suitable transfer powers. In [19], the author used an MRC for an implantable medical unit to recharge a battery with a distance between the receiver and transmitter coils. In [20], a researcher used an MRC for the implanted medical system to charge the battery through an air gap and receive the battery state using the ZigBee protocol. Another study used WPT to operate a temperature sensor implanted inside a rabbit's body [21]. Other authors have used WPT with MRC to enhance power transfer and efficiency at different air-gap distances between coils to supply medical devices. In addition, studies [22-25] have used statistical analyses to calculate the accuracy of measuring biomedical parameters, such as temperature and heart rate. The contributions of this study are as follows.

1. MRC-WPT system was practically implemented to provide sufficient power for heartrate measurements in humans;

2. The accuracy of patients' heart-rate measurements was validated relative to the benchmark (BM) device;

3. Power transfer and efficiency were investigated for the proposed MRC-WPT system;

4. The functionalities of the heart-rate measurement device were achieved based on MRC-WPT;

5. The heart-rate measurement accuracy, and Transfer efficiency, and power transfer of the MRC-WPT are compared to those reported in related works to confirm its achievement.

The remainder of the paper is structured as follows. Previous studies related to the performance metrics of power transfer and efficiency are described in Section 2. Section 3 presents the design of the MRC-WPT and explains the experimental configuration of the MRC-WPT technique. In addition, the Transfer efficiency, power transfer, and statistical analyses are provided and discussed in this section. The results and discussion are presented in Section 4. The results of the MRC-WPT system were compared with previous work in terms of relevant parameters in Section 5. Finally, conclusions and future recommendations are introduced in Section 6.

\section{Related Works}

Recently, scholars have considered using WPT in electronics and different medical applications. Xing et al. [11] designed and executed a WPT method utilizing IC for implantable medical equipment such as retinal prostheses and neural recordings without the use of a battery. The functioning frequency of the oscillator was $13.56 \mathrm{MHz}$. The system comprises a receiving coil, transmitting coil, rectifier, and resistive load. Practical results illustrated that the power transfer during loading was $102 \mathrm{~mW}$. In addition, the maximum transfer power efficiency was $92.6 \%$ at an air gap of $1 \mathrm{~cm}$. The main advantages of the proposed technique were low cost; however, the transfer distance was short. Jiang et al. [16] implemented WPT employing an MRC to enhance the transfer power efficiency between coils for small household appliances and implanted devices with a $6.78 \mathrm{MHz}$ operating frequency. The system included a power source and coils in the receiver and transmitter. Practical outcomes showed that the power transfer was $0.001 \mathrm{~W}$ when using $R L=50 \Omega$ at a $4 \mathrm{~cm}$ distance. Once again, the main advantage of the system was low cost; however, the system was prohibitively large.

Mohamadi et al. [17] executed WPT utilizing MRC to recharge a battery for implanted medical devices, e.g., artificial hearts, monitoring devices, left-ventricular assistance devices, and drug infusion. The system included ZVS, a transmitter coil, a control switch, a receiver coil, a resonator capacitor, and an advanced RISC AVR microcontroller. The 
operating frequency was controllable to study transfer power. The results showed that power in the receiver coil decreased at large transfer distances. The output power during loading was $1.5 \mathrm{~W}$. The system had a long duty cycle for the battery and improved Transfer efficiency. However, it was large in size and required simulation using the Proteus software. Zhang et al. [18] designed a WPT technique employing MRC for implantable devices and medical sensors. The WPT technique included a power source, coils in the receiver and transmitter, and a load; $7 \mathrm{MHz}$ was the operating frequency for the system, which was implemented on pigs. The proposed system was implanted $3.5 \mathrm{~cm}$ under pigskin. Then, the Transfer efficiency was found to be $22.3 \%$ at $9 \mathrm{~cm}$. In addition, the measurement accuracy was $70 \%$. This system may work well over short distances; however, it was large, unstable, and suffered from power leakage.

Campi et al. [19] suggested a WPT system employing MRC to recharge batteries for an implantable medical gadget, such as an LVAD; $300 \mathrm{kHz}$ was the operating frequency for the system, which included transmitting and receiving coils. The results indicated that the transfer power efficiency was $47 \%$ and $7 \%$ at 3 and $6 \mathrm{~cm}$, respectively. In addition, the measurement accuracy was 55\%. The advantage of this system was that it was portable. However, it only worked over a short distance. Gore et al. [20] implemented WPT by using MRC to recharge a battery for operation in implantable medical devices such as PMs; $300 \mathrm{kHz}$ and $13.56 \mathrm{MHz}$ were operating frequencies for the system. The system included coils in the transmitter and receiver, a bridge rectifier, a microcontroller based on the 16F877A chip, the ZigBee communication model, an LCD, and a battery. The results showed that, when the battery was fully charged, ZigBee sent the battery condition to the power transmitter and the relay turns off WPT. The system then displays the battery voltage on the LCD. The utmost transfer voltage of the proposed system did not exceed $11 \mathrm{~V}$ at an air gap of $15 \mathrm{~cm}$. In addition, the measurement accuracy was $95 \%$. The main advantage of this device was that it was wearable and safe.

Hussain et al. [21] presented a WPT system for medically implanted devices such as temperature sensors; $67 \mathrm{kHz}$ was the working frequency of the proposed technique. The proposed technique comprised a power source, coils in the receiver and transmitter, a rectifier, and the load. The load included a temperature sensor, nRF24L01 module, and Arduino microcontroller. The experiment was conducted on rabbits. The results revealed that the power transfer and transfer power efficiency were $1 \mathrm{~W}$ and $15 \%$ at $1 \mathrm{~cm}$, respectively. In addition, the measurement accuracy was $96 \%$. The advantage of the system was that it could operate without a battery. However, the system worked only over a short distance. Zhang et al. [22] presented a WPT system that relied on MRC to study the power transfer and efficiency in the air gap using a spiral coil. The system comprised a power source and coils in the source and load. The experimental results revealed that the voltage increased with mutual inductance. In addition, the power transfer and efficiency were $14 \mathrm{~W}$ and $67 \%$ in a $4-\mathrm{cm}$ distance, respectively. Moreover, the measurement accuracy was variable based on mutual inductances about $88 \%$ in a $4-\mathrm{cm}$ air gap. The advantage of this system was that it could be used in medical equipment. However, it suffered from coupling losses and did not implement statistical analyses.

Campi et al. [24] executed WPT-based MRC in functional medical gadgets; the functioning frequency for the MRC technique was $4 \mathrm{MHz}$. The MRC technique included a coil in the transmitter, destination and load. The outcomes showed that the transfer voltage, power, and efficiency in the Rx coil were $4 \mathrm{~V}, 10 \mathrm{~mW}$, and $1 \%$, respectively, at $3 \mathrm{~cm}$. In addition, the measurement accuracy was $94 \%$. The main advantage of this technique was its low cost. However, it could only operate at a short distance. Zakaria et al. [25] presented a WPT-based MRC technique for smart-home applications and medical systems; the working frequency for the MRC was 30-81 MHz. The MRC system included coils in the receiver and transmitter, a bridge, and a load. Two techniques were investigated in the experiment; the results showed that the power Transfer efficiency was $21.74 \%$ and $14.76 \%$ at $5 \mathrm{~cm}$ in case 1 and case 2, respectively. In addition, the measurement accuracy was $70 \%$. This system was low in cost but large in size. Pavani et al. [23] implemented WPT using MRC to develop 
the power Transfer efficiency between coils for small household appliances and implanted devices; $300 \mathrm{kHz}$ was the operating frequency for the system. The system included a power source and coils in the receiver and transmitter. The practical outcomes showed that the power Transfer efficiency was $0.75 \%$ when using a $20 \Omega$ resistive load (RL) with mutual inductances of $5.5 \mu \mathrm{H}$ at $4 \mathrm{~cm}$. Besides, the accuracy of the measurements was $94 \%$. Again, this system was low in cost but large in size. The performance metrics for certain WPT systems are summarized in Table 1 in terms of their objective, operating frequency, application, measurement accuracy, transfer distance, efficiency, and power.

Table 1. Performance metrics of previous studied.

\begin{tabular}{|c|c|c|c|c|c|c|c|}
\hline $\begin{array}{l}\text { Ref/MRC } \\
\text { Technique }\end{array}$ & Objective & $\begin{array}{c}\text { Frequency } \\
\text { (MHz) }\end{array}$ & Application & $\begin{array}{c}\text { Accuracy } \\
(\%)\end{array}$ & $\begin{array}{l}\text { Distance } \\
\text { (cm) }\end{array}$ & $\begin{array}{c}\text { Efficiency } \\
(\%)\end{array}$ & $\begin{array}{c}\text { Power } \\
\text { (W) }\end{array}$ \\
\hline [17] & OTE & 0.00988 & LVAD & $\mathrm{N} / \mathrm{A}$ & $\mathrm{N} / \mathrm{A}$ & $\mathrm{N} / \mathrm{A}$ & 1.5 \\
\hline [18] & OTE & 7 & ID & 70 & 9 & 22.3 & $\mathrm{~N} / \mathrm{A}$ \\
\hline [19] & OTDE & 0.3 & ICD & 55 & 6 & 7 & 1 \\
\hline [20] & BT ch. & $0.3-13.56$ & PM & 95 & 10 & $\mathrm{~N} / \mathrm{A}$ & 0.0004 \\
\hline [21] & OTDE & 0.067 & TS & 96 & 3 & 1 & 0.05 \\
\hline [22] & OTDE & $\mathrm{N} / \mathrm{A}$ & MS & 88 & 4 & 67 & 14 \\
\hline [24] & OTE & 4 & MD & 94 & 3 & 1 & 0.01 \\
\hline [25] & OTE & 30 & MS & 70 & 5 & 14.7 & $\mathrm{~N} / \mathrm{A}$ \\
\hline [23] & OTE & 0.3 & ID & 94 & 4 & 0.75 & $\mathrm{~N} / \mathrm{A}$ \\
\hline
\end{tabular}

N/A: not available; OTE: optimization Transfer efficiency; BT ch.: battery charger; OTDE: optimize transfer distance and efficiency; ID: implantable devices; ICD: implantable cardioverter defibrillators; TS: a temperature sensor; MD: medical sensor.

\section{Research Methodology}

Due to the problem of limited battery capacity of medical devices limiting their functionality, the materials and methods presented in this work aim to design and implement an MRC-WPT-based WHR measurement system to solve the current problem in such systems. The proposed MRC-WPT can be practically implemented and tested by adopting four coils topologies to select the best topology that can give higher performance in terms of power transfer and efficiency. In addition, the functionalities of the WHR measurement can be achieved using MRC-WPT in which the MRC-WPT can provide sufficient power for the WHR measurements unit. The measurements accuracy of the WHR can be verified relative to the BM device (HANSA) (HANSA Medical Germany, Frankfurt, Germany). The aforementioned materials and methods can be performed by adopting the following.

First, the designed parameters and equivalent circuit of spiral-spider coil were introduced. Four types of coils topologies; spiral-spiral, spider-spider, spider-spiral, and spiral-spider were designed to select the best topology that can give higher performance in terms of power transfer and efficiency. In addition, the equivalent circuit of the wireless power transfer system was described.

Second, simple mathematical models for output power in watt and efficiency in percentage were presented to evaluate the transfer performance of the MRC-WPT.

Third, the MRC-WPT model for powering the WHR monitoring system was presented. The MRC-WPT contains three units: a transmitter, receiver, and monitoring unit. The receiver unit is in charge of powering the WHR sensor.

Fourth, after completing the circuit design, four experiments were configured and conducted to test the performance of the spiral and spider coil using four topologies. The experimental protocol and test parameters adopted in experiments were described in detail. One topology can be adopted for supplying the WHR sensor by power according to the best performance of power transfer and efficiency. The structure of the methodology is presented in Sections 3.1-3.6 as follows.

Fifth, analysis and validation of the WHR measurement were presented based on collected data from both proposed WHR and BM systems. In both systems, 100 samples 
(one sample every minute) were gathered simultaneously to facilitate the comparison between the two systems.

Finally, the measurement accuracy of WHR was evaluated relative to the BM device using a simple mathematical formula.

\subsection{Coils Design}

In this paper, we wrapped two coils separately on the wooden base, relying on hand skill to construct spiral and spider coils. The coils were looped around a screw fixed on a wooden base. The coils were then bonded employing glue. Based on these two coils, four topologies were experimentally tested for the transmitter and receiver coils, as shown in Figure 1. To investigate the performance of the proposed topologies (i.e., spiral-spiral, spider-spider, spider-spiral, and spiral-spider) at $50 \Omega$ resistive load, the output power, Transfer efficiency, output voltage (load voltage), and output current (load current) can be extracted and compared later on in the result section. In this research, the spider coil was designed by using 150 turns while the spiral coil employed 91 turns. However, a trade-off between cost and performance in terms of power transfer and efficiency was taken into consideration when designing coils. Therefore, spiral-spider coils topology was selected due it conveying higher transfer power and efficiency than other topologies. In addition, spiral and spider coils are similar to the circular coil shape where their mutual inductances are higher than that of the square coil because they have a bigger overlapping area [26]. Accordingly, the spiral and spider coils were adopted, and the square coil design was excluded from the current research.
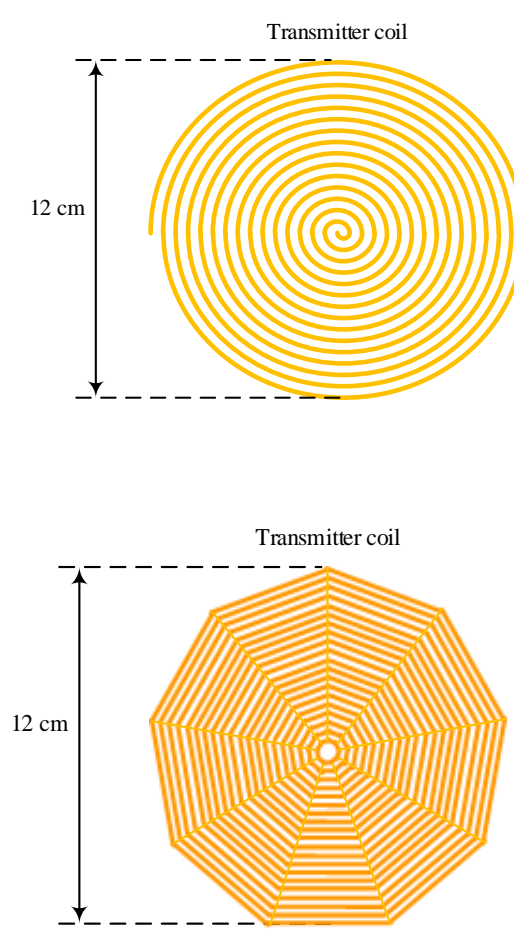

Figure 1. Cont.

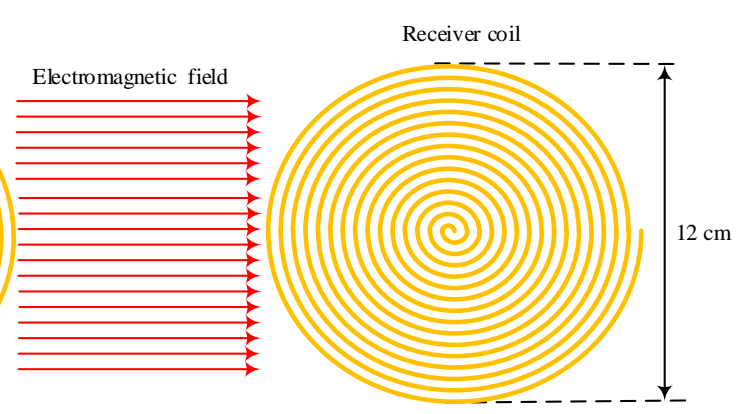

(a)

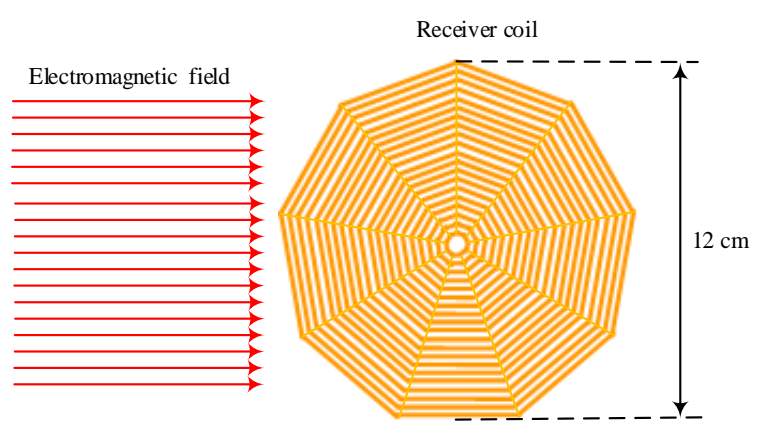

(b) 

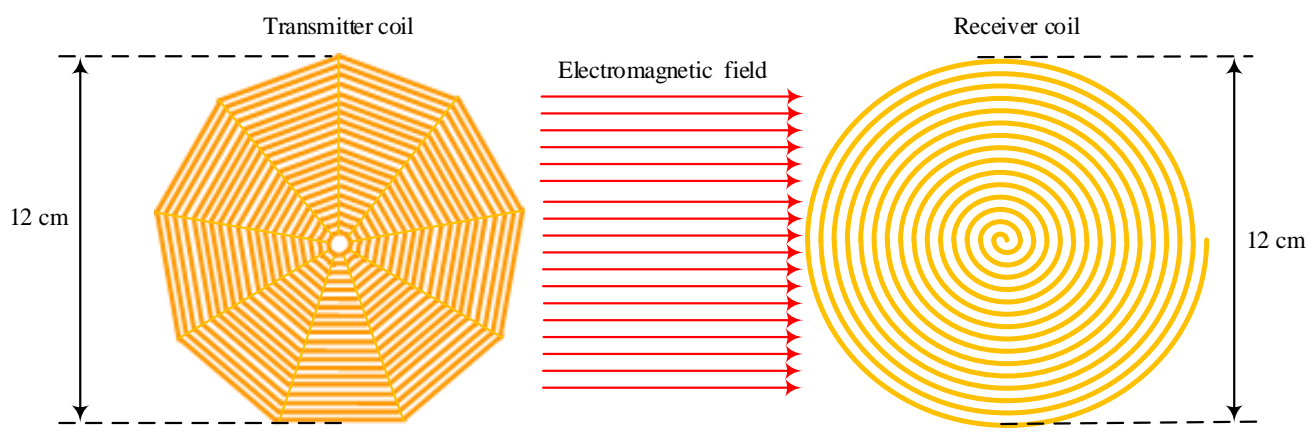

(c)
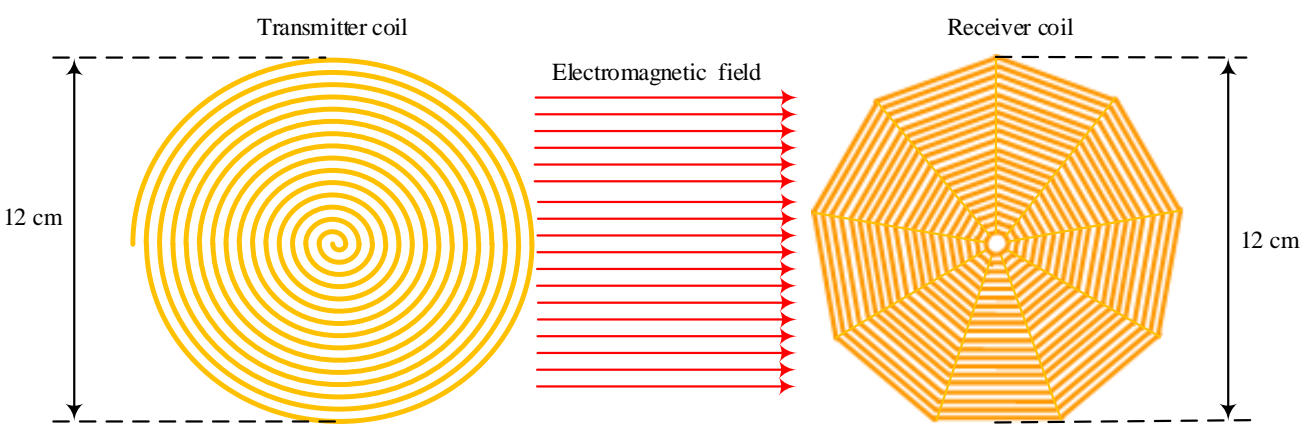

(d)

Figure 1. Coils topologies (a) spiral-spiral, (b) spider-spider, (c) spider-spiral, and (d) spiral-spider.

The spiral and spider coils parameters are presented in Table 2. The oscillator in the transmitter unit generates a frequency of $13.5 \mathrm{kHz}$, and the inductance of the spiral and spider coils are 695 and $720 \mu \mathrm{H}$. Therefore, the resonance capacitors can be connected in parallel with the coils, can be computed based on Equation (1) to transfer maximum power from the transmitter coil to the receiver coil at resonant frequency $\left(f_{0}\right)$.

$$
f_{0}=\frac{1}{2 \pi \sqrt{L_{T} C_{T}}}=\frac{1}{2 \pi \sqrt{L_{R} C_{R}}}
$$

where $L_{T}$ and $C_{T}$ and $L_{R}$ and $C_{R}$ are the coil inductance and resonance capacitors of the spiral and spider coils, respectively.

Table 2. The adopted parameters of spiral and spider coils.

\begin{tabular}{ccc}
\hline Parameter & Spiral Coil & Spider Coil \\
\hline Conductor diameter $(\mathrm{mm})$ & $0.724(21 \mathrm{AWG})$ & $0.724(21 \mathrm{AWG})$ \\
Coil outer diameter $(\mathrm{cm})$ & 12 & 12 \\
No. of turns & 91 & 150 \\
Inductance $(\mu \mathrm{H})$ & 695 & 720 \\
Resonance capacitor $(\mathrm{nF})$ & 200 & 190 \\
Operating frequency $(\mathrm{kHz})$ & 13.5 & 13.5 \\
\hline
\end{tabular}

Accordingly, the resonance capacitors are 200 and $190 \mathrm{nF}$ for $C_{T}$ and $C_{R}$, as listed in Table 2, respectively. The equivalent circuit of the wireless power transfer system is shown in Figure 2. 


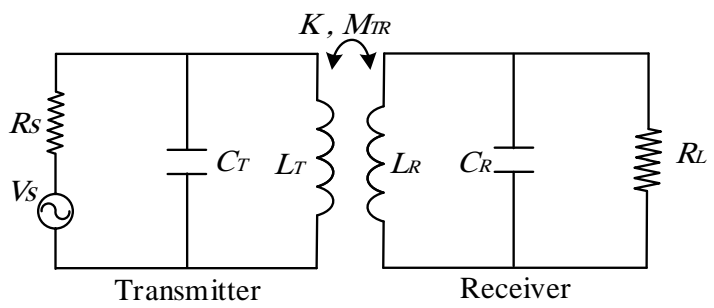

Figure 2. Equivalent circuit of the wireless power transfer system.

\subsection{Performance of Transfer Power and Efficiency}

Battery-charging systems based on the WPT technique should be effective regarding power Transfer efficiency and output power [27]. Therefore, it is important to examine the efficiency and power transfer of the proposed MRC-WPT-based WHR monitoring system. The output power was computed using Equation (2) [21], while the efficiency was calculated using Equation (3) [1,21].

$$
\begin{gathered}
\text { Output power }=\text { voltage } \times \text { current } \\
\text { Transfer efficiency }(\eta) \%=\frac{\text { output power }}{\text { iutput power }} \times 100 \%
\end{gathered}
$$

where the voltage and current in Equation (2) are the load voltage and load current, respectively.

\subsection{System Model}

The schematic diagram of the MRC-WPT-based heart-rate monitoring system is shown in Figure 3. The MRC-WPT contains three units: a transmitter, receiver, and observing unit. The transmitter units consist of a power part, oscillator circuit, and source coil $\left(T_{x c}\right)$, which is a spiral. The receiver unit consists of two components, the power and measurement components. The power component contains the receiver coil $\left(\mathrm{R}_{\mathrm{xc}}\right)$, which is a spider coil; resonant capacitor; and bridge rectifier. The measurement component comprises a microcontroller-based Arduino Nano platform, a heart-rate sensor, and a wireless communication based-nRF24L01 chip. The measurement component measures the heart rate of the patient and transmits the data to a monitoring unit (which includes the Arduino Mega-2560 platform, nRF24L01, and computer), to be monitored using a laptop in real-time.

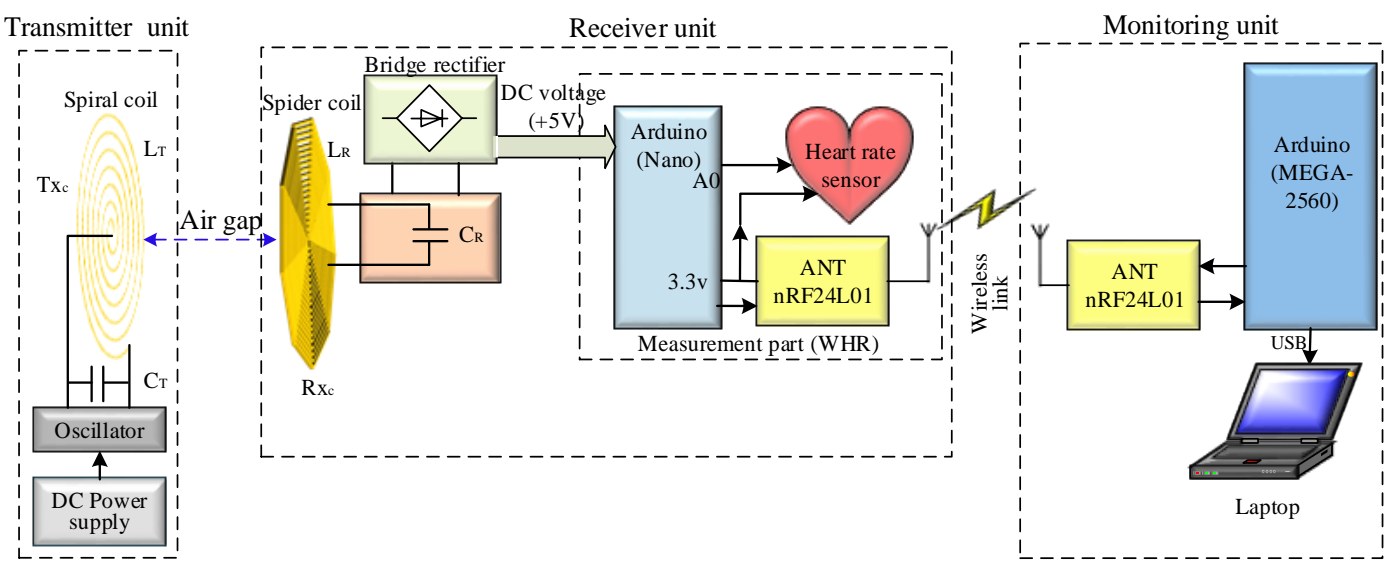

Figure 3. Block diagram of the MRC-WPT-based WHR monitoring system.

The MRC theory is constructed on the air gap between the transmitter and receiver coils. The resonator capacitors in the transmitter and receiver units are coupled in parallel with the $\mathrm{L}_{\mathrm{T}}$ and $\mathrm{L}_{\mathrm{R}}$ coils, respectively. The parallel-to-parallel compensation topology was chosen due to (i) facilitating higher power transfer between the two coils [28,29], (ii) it has 
a better performance than series-to-series topology in low power applications [30], where the proposed WHR consumes low power $(\approx 0.166 \mathrm{~W})$ and (iii) the ZVS oscillator (Xiongfaic Weiye Electronics Co., Ltd., Shenzhen, China) existing in our lab is restricted to the parallel topology and low oscillator frequency. The low frequency is suitable for using with parallel resonator topology, where the parasitic losses are reduced, while the series topology is more suitable for employing with high frequency [31].

The rectifier uses a Schottky diode (module SR260) (Won-Top Electronics Co., Ltd., Kaohsiung City, Taiwan) to adapt AC to DC [32], because these diodes have a low voltage drop, approximately $0.18 \mathrm{~V}$, to guarantee that most of the voltage is conveyed to the load. The measurement part in the receiver unit measures the patient's heart rate and transmits the data to the monitoring unit via the nRF24L01 (Kuongshun Electronic Ltd., Shenzhen, China). The power source supplied adequate power to operate the measurement component, which depended on a microcontroller (Arduino Nano) (Arduino, Italy) to supply a sensor and nRF24L01 with the operating voltage. The laptop in the monitoring unit is used to deliver the power to the microcontroller (Arduino Mega 2560) (Arduino, Italy), as well as surveillance the heart rate of the patient. Then, the microcontroller supplied the nRF24L01 with a sufficient operating voltage of $3.3 \mathrm{~V}$. The nRF24L01 wireless module then received the heart rate data from the measurement component. Next, it sent the data to the Mega 2560 microcontroller to be displayed on the monitor of the laptop.

\subsection{Experiment Configuration}

In this experiment, two types of coils were used: spiral and spider coils. Four experiments were conducted for four topologies (spiral-spiral, spider-spider, spider-spiral, and spiral-spider) to test the performance of the coils. One topology can be considered for supplying the WHR sensor by power according to the best performance. The experiment depicted in Figure 4a was conducted to examine the performance of the MRC-WPT-based WHR monitoring system regarding power transfer, power Transfer efficiency, the air gap between coils, DC output voltage, and WHR measurement accuracy. The oscillator model (ZVS) in the transmitter unit functions at a frequency of $13.5 \mathrm{kHz}$. Based on this frequency, the coil in the transmitter unit produces an electromagnetic field which causes an induced current in the receiver coil. In this context, the power is transferred from the transmitter coil to the receiver coil, which in turn, can supply the measurement component by power. This MRC-WPT-based WHR measurement was used on a medical device to send heartbeat information to the central station or monitoring unit employing the nRF24L01 wireless protocol. The Arduino Nano was utilized in the measurement component owing to its small size and low cost [33].

The nRF24L01 wireless protocol was employed in the transmitter and monitoring units owing to its ultra-low power, cost-effectiveness, low weight, suitable transmission distance up to $100 \mathrm{~m}$, and operation on the free-license radio frequency band of $2.4 \mathrm{GHz}[34,35]$. The nRF24L01 module has various uses, for example, wireless peripherals for computers, data transmission, wireless sensors, and ultra-low power sensor systems. The adopted heart rate sensor has a low cost, low power drain, and small size, with a diameter of $16 \mathrm{~mm}[36,37]$. To minimize the power drain of the heart rate sensor, a low supply voltage was adopted $(3.3 \mathrm{~V})$ for its functioning. The transmitter coil was permanently kept perpendicular to the earth. Whereas the receiver coil was positioned in front of the transmitter coil with the right alignment, the highest power transfer was guaranteed. Thus, an AC voltage was produced in the receiver circuit based on the receiver coil and resonant capacitor. The DC voltage can be obtained from AC voltage using a bridge rectifier (based on Schottky-diodes), which is low in cost and small in size.

The DC output voltage of the system was measured and recorded in $1 \mathrm{~cm}$ horizontal steps between transmitter and receiver coils until a $25 \mathrm{~cm}$ distance was reached. Hence, the MRC-WPT-based WHR system was tested under alignment conditions. The misalignment conditions pose several challenges in WPT, for example, it changes the system parameters such as mutual and self-inductances, which lead to system instability, reduce transfer 
power and efficiency, and increase power losses owing to the detuned operation [38]. Therefore, the misalignment conditions were not considered in this study and was left for future work.

The MRC-WPT was tested at a $50 \Omega$ resistive load. Thus, the output power and system Transfer efficiency can be computed for those distances. It was found that, at a $20 \mathrm{~cm}$ distance, the measurement unit can be operated accurately without any failure in the supply voltage. Therefore, the WHR component can accurately transmit data to the monitoring unit, as shown in Figure 4b. The experimental protocol and the test parameters adopted in the current experiment are illustrated in Table 3.

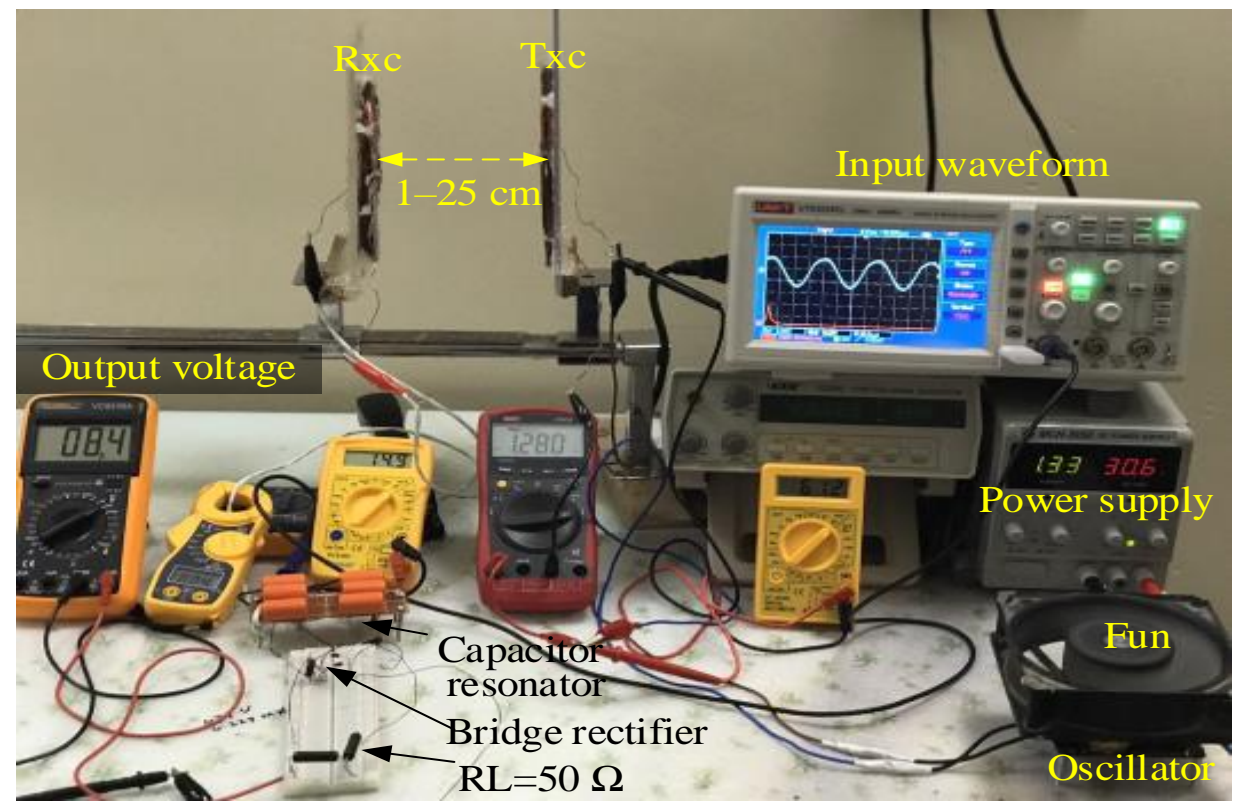

(a)

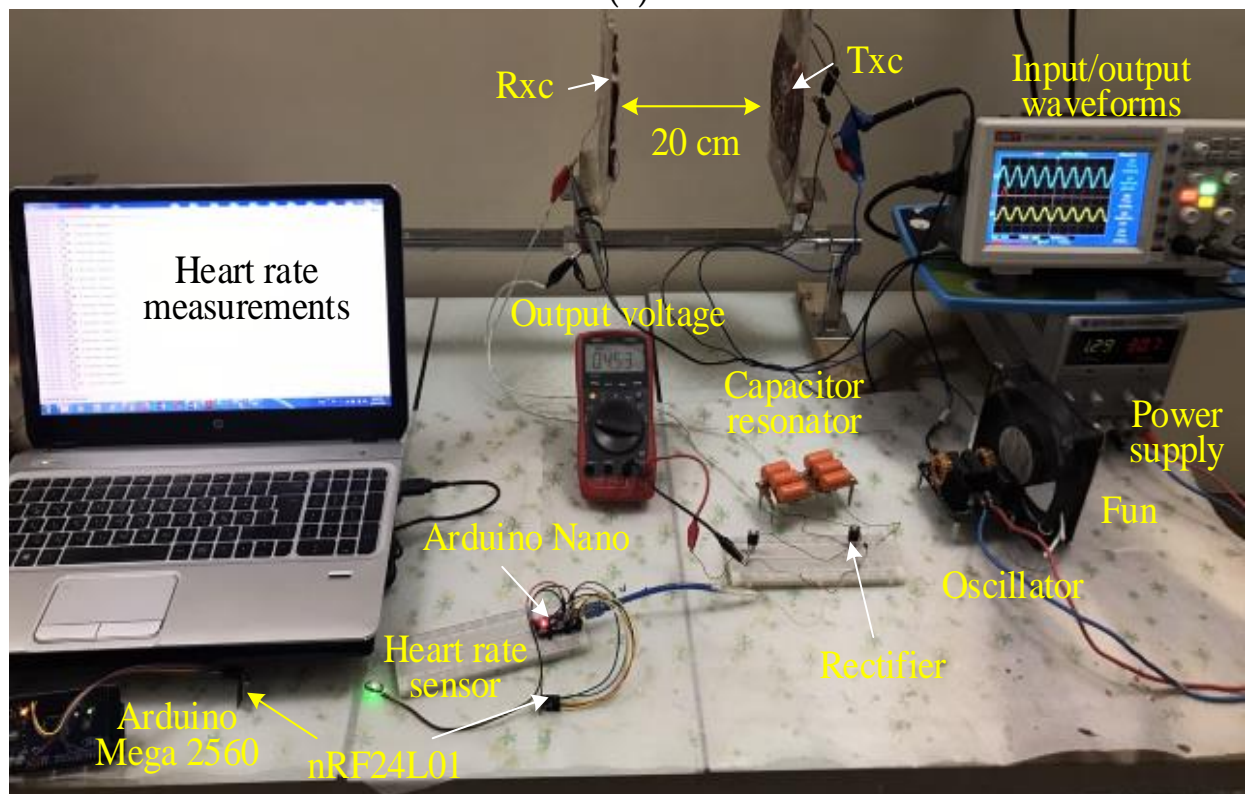

(b)

Figure 4. The experiment of (a) explores the key parameters of the MRC-WPT-based WHR monitoring system and (b) WHR measurement at $20 \mathrm{~cm}$. 
Table 3. Experimental protocol and the test parameters adopted in the current experiment.

\begin{tabular}{|c|c|}
\hline Parameter & Description \\
\hline Coils & spiral and spider \\
\hline Topologies & spiral-spiral, spider-spider, spider-spiral, spiral-spider \\
\hline Oscillator circuit & ZVS $1000 \mathrm{~W} / 20 \mathrm{~A}$ with a DC input voltage of $12-30 \mathrm{~V}$ \\
\hline Resonance frequency of the oscillator & $13.5 \mathrm{kHz}$ \\
\hline Bridge rectifier & Schottky diodes (module SR260) \\
\hline Heart rate sensor & Pulse sensor (SEN-11574) \\
\hline Processing & $\begin{array}{ll}- & \text { Arduino Nano microcontroller (for WHR sensor) } \\
\text { - } & \text { Mega } 2560 \text { microcontroller (for Monitoring unit) }\end{array}$ \\
\hline Wireless protocol (nRF24L01) [35] & $\begin{array}{ll}\text { - } & \text { Operating frequency: } 2.4 \mathrm{GHz} \\
\text { - } & \text { Operating voltage: } 3.3 \mathrm{~V} \\
\text { - } & \text { Current consumption: } 11.3 \mathrm{~mA} \\
\text { - } & \text { Data rate: up to } 2 \mathrm{Mbps} \\
\text { - } & \text { Communication distance: up to } 1000 \mathrm{~m} \text { for nRF24L01+ }\end{array}$ \\
\hline $\begin{array}{l}\text { Laptop } \\
\text { Tested distance }\end{array}$ & $\begin{array}{l}\text { Monitoring the heart rate measurements based on Arduino IDE software } \\
1-25 \mathrm{~cm}\end{array}$ \\
\hline Tested devices & $\begin{array}{ll}\text { - } & \text { Digital multimeter } \\
\text { - } & \text { Digital storage oscilloscope } \\
\text { - } & \text { LAB power supply (0-30 V/5 Amp.) }\end{array}$ \\
\hline
\end{tabular}

\subsection{Analysis and Validation of Collected Data}

Once the experiment setup presented in Section 3.4 is completed, the MRC-WPT starts to supply the WHR system by power. The pulse heart rate sensor connected to the patient's finger measures and sends the heart rate measurements to the Arduino Nano microcontroller in the measurement parts. The microcontroller transmits the measured data to the monitoring unit via nRF24L01 wireless protocol. The heart rate data are received by the nRF24L01 in the monitoring unit and recorded by Arduino Mega 2560 microcontroller. To validate the measurement accuracy of the WHR system, the BM device (Figure 5) is used to facilitate a comparison between WHR and BM systems. The BM is connected to the patient's upper arm to measure the patient's heart rate. In both systems, the heart rate data (100 samples, one sample every minute) are collected simultaneously, as illustrated in Figure 6.

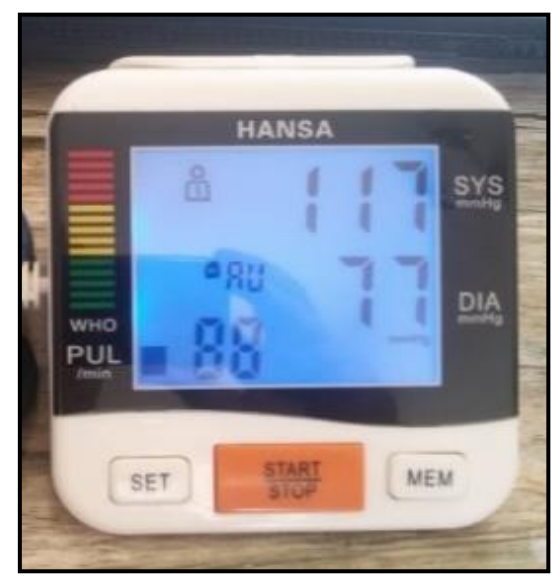

Figure 5. The BM device. 


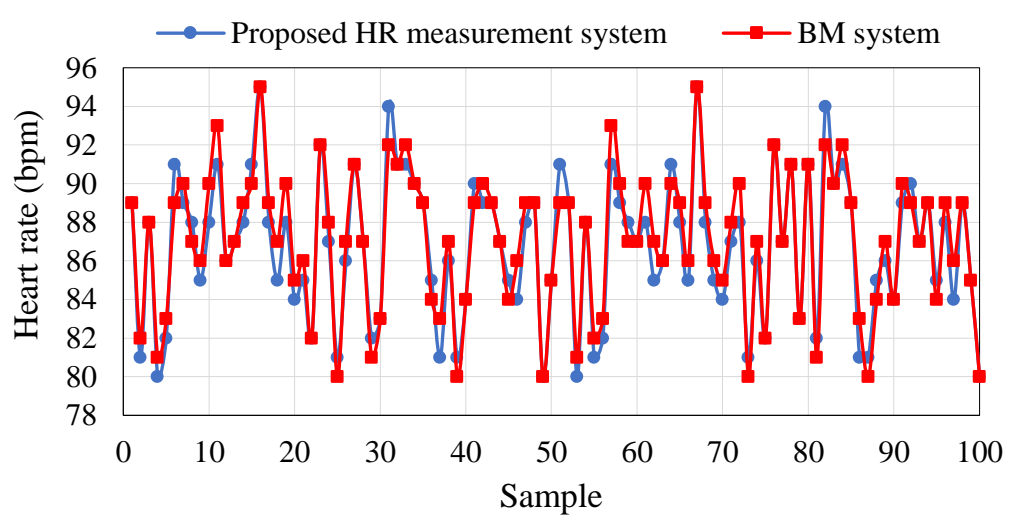

Figure 6. Measurement of WHR and BM systems.

The measurements of the BM device are recorded manually due to the automatic restricted access to the data, whereas the data of the WHR system are received by the monitoring unit and analyzed using Arduino Mega 2560 microcontroller. Then, the data acquired by Arduino Mega 2560 microcontroller are sent to the laptop through a USB port at a baud rate of 115,200 bps and are documented in Parallax Microcontroller Data Acquisition (PLX-DAQ) for Excel [39]. The PLX-DAQ can plot data on the $x$ - and y-axes in real-time. The $x$-axis demonstrates the number of samples of the patient's heart rate. In contrast, the $y$-axis shows the heart rate measurement for the WHR and BM systems in beats per minute (bpm). The blue line denotes the heart-rate measurement obtained from the WHR system, while the red line denotes the measurement obtained from the BM device.

It is found that the heart rate measurements exhibit slight variances between the BM system and the WHR system. To check the heart rate obtained using the WHR measurement system, statistical analyses were conducted, including error tests, measurement accuracy, the Bland-Altman test, and histograms, as will be introduced later, in Section 4.2. The Bland-Altman test is a method of statistical analysis [40] commonly considered in medical statistics to indicate the possibility of similarities or differences in agreement between two systems. In this paper, the Bland-Altman test was adopted to examine the similarity between the WHR measurement system and the BM device. The histogram is the most common method for graphical feature representation in terms of frequency distribution. Therefore, it can be used to show peak scores of the two systems.

\subsection{Measurement Accuracy of WHR}

The performance metrics of the WHR system can be evaluated in terms of measurement accuracy based on the collected data from WHR and BM systems. The WHR measurement accuracy can be calculated based on Equation (4) [41]. The accuracy denotes the overall ability of the WHR in measuring the heart rate, which closely matches the measurements of the BM system.

$$
\operatorname{Accuracy}(\%)=\frac{\frac{1}{N} \sum_{i=1}^{N} x_{i}}{\frac{1}{N} \sum_{i=1}^{N} y_{i}} \times 100 \%
$$

where $N$ is the samples of the measurement; 100 samples were considered in this experiment. $x_{i}$ and $y_{i}$ denote the WHR measurement for each sample for the proposed system and BM system, respectively.

\section{Results and Discussion}

\subsection{Result of Transfer Power and Efficiency}

The measured transfer efficiencies and powers at a resistive load of $50 \Omega$ are illustrated in Figure 7. The output power and efficiency were measured at the different distances 
between $T_{x c}$ and $R_{x c}$ every $1 \mathrm{~cm}$ step until $25 \mathrm{~cm}$. The transfer air gaps, in centimeters, are drawn on the $x$-axis; the output power, in watts (W), and efficiency in percentage, are drawn on the right and left sides of the $y$-axis, respectively. The output power and Transfer efficiency were examined for four topologies as shown in Figure 7a-d for spiral-spiral, spider-spider, spider-spiral, and spiral-spider topologies, respectively. Figure $7 \mathrm{a}-\mathrm{d}$ shows that the Transfer efficiency and output power were $31.11 \%$ and $6.88 \mathrm{~W}, 38.18 \%$ and $4.08 \mathrm{~W}, 70.816 \%$ and $19.62 \mathrm{~W}$, and $86.21 \%$ and $23.45 \mathrm{~W}$, respectively, in a $4 \mathrm{~cm}$ air gap. While Figure $7 \mathrm{a}-\mathrm{d}$ shows that the Transfer efficiency and output power were $20.02 \%$ and $1.71 \mathrm{~W}, 14.36 \%$ and $1.32 \mathrm{~W}$, $31.39 \%$ and $4.01 \mathrm{~W}$, and $40.36 \%$ and $4.47 \mathrm{~W}$, respectively, in a $7-\mathrm{cm}$ air gap. However, the efficiency and output power decreased to $2.09 \%$ and $0.207 \mathrm{~W}, 0.46 \%$ and $0.202 \mathrm{~W}, 1.68 \%$ and $0.513 \mathrm{~W}$, and $2.42 \%$ and $0.55 \mathrm{~W}$ for Figure $7 \mathrm{a}-\mathrm{d}$, respectively, in a 12-cm air gap between transmitter and receiver coils. Moreover, the values of Transfer efficiency and output power further decreased to less than $0.52 \%$ and $0.189 \mathrm{~W}$ for spiral-spider topology, respectively, in $15 \mathrm{~cm}$ air gaps and higher. As a result, the spiral-spider outperformed other topologies in terms of Transfer efficiency and output power.

Figure 8a-d illustrated the performance of the proposed topologies including output power, Transfer efficiency, output voltage (load voltage), and output current (load current) at $50 \Omega$ resistive load, respectively. The figures revealed that the spiral-spider topology beats other topologies in terms of output power, Transfer efficiency, output voltage, and output current, as highlighted in the blue line in all figures. Figure 8a-d shows that the output power, Transfer efficiency, output voltage, and output current were $0.55 \mathrm{~W}, 2.24 \%, 5 \mathrm{~V}$, and $110 \mathrm{~mA}$, respectively, in a $12 \mathrm{~cm}$ air gap.



(a) Spiral-Spiral

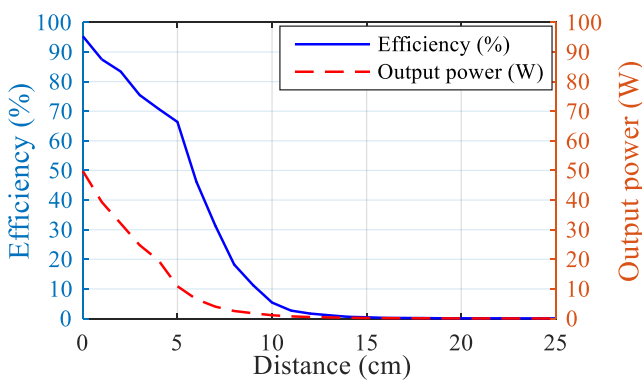

(c) Spider-Spiral

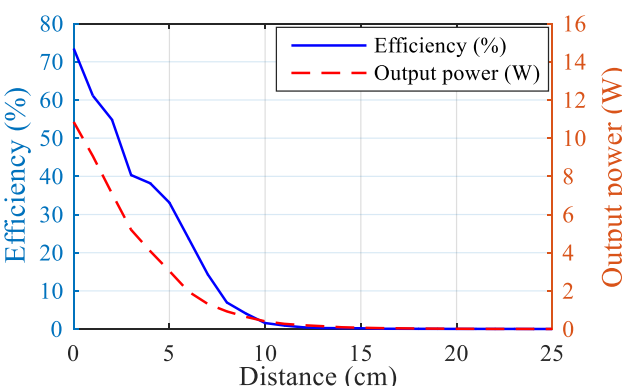

(b) Spider-Spider



(d)Spiral-Spider

Figure 7. Transfer power and efficiency for different topologies: (a) spiral-spiral, (b) spider-spider, (c) spider-spiral, and (d) spiral-spider at $50 \Omega$ resistive load. 


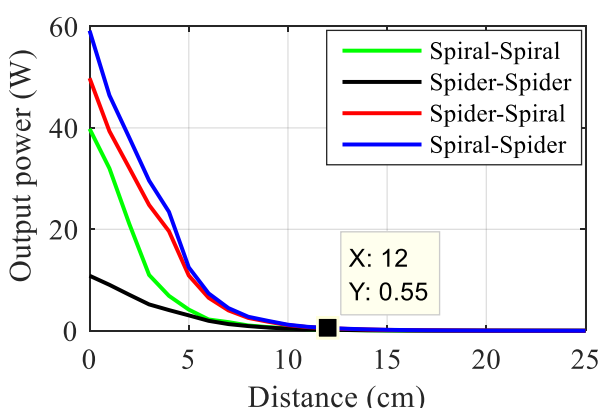

(a)

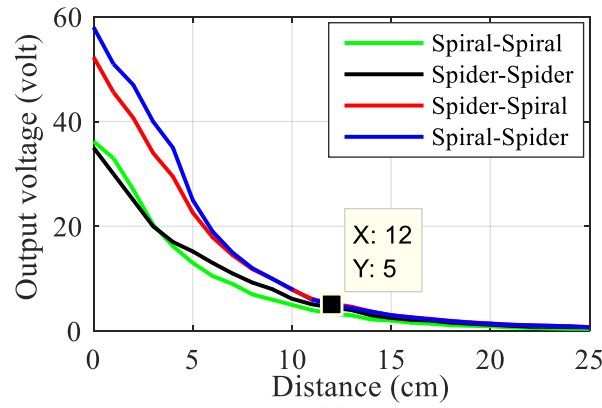

(c)

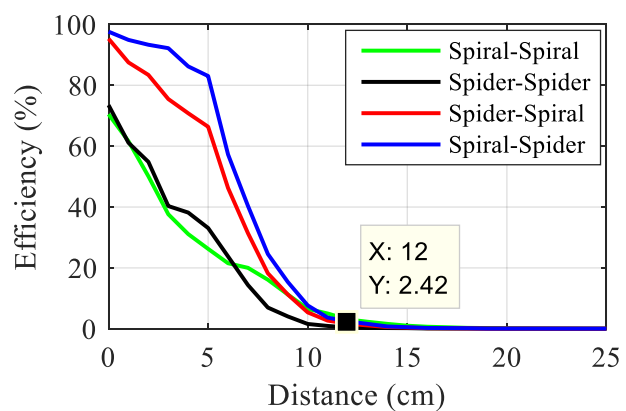

(b)



(d)

Figure 8. Performance of the proposed topologies: (a) output power, (b) Transfer efficiency, (c) output voltage (load voltage), and (d) output current (load current) at $50 \Omega$ resistive load.

All of the components of the measurement system consume approximately $33.2 \mathrm{~mA}$. The current consumptions are $19 \mathrm{~mA}$ [42], $12 \mathrm{~mA}$ [43], and $2.2 \mathrm{~mA}$ [44] for the nRF24L01, Arduino nano, and heart rate sensor-based pulse sensor (SEN-11574), respectively. In this context, an output power of $0.166 \mathrm{~W}(33.2 \mathrm{~mA} \times 5 \mathrm{~V}$ (operating voltage) ) is sufficient for operating the measurement component of the proposed system. Therefore, the measurement component can work properly without system failure between 1 and $15 \mathrm{~cm}$ air gaps where the generated output power, output voltage, and output current were more than the required values of the measurement unit. However, the system fails to operate beyond $15 \mathrm{~cm}$. The measurement unit was tested using the output voltage and current of the MRC-WPT to confirm its measurement performance, as will be seen in the next section.

\subsection{Measurement Validation of WHR}

The measurement data for patient WHR can be validated relative to the BM (HANSA device previously shown in Figure 5) based on several statistical analyses as follows:

\subsubsection{Error Test and Accuracy}

Figure 9 presents the errors and average error/mean absolute error (MAE) of the proposed HR system relative to the BM device. The $x$-axis indicates the number of samples, while the $y$-axis denotes the absolute error and MAE. The figure shows that the heart rate error varies between 0 and $2 \mathrm{bpm}$. Most of the errors were less than or equal to $1 \mathrm{bpm}$, while certain error samples were less than or equal to $2 \mathrm{bpm}$. However, relative to the MB device, the obtained MAE is 0.78 for the proposed WHR measurement system. To compute the WHR measurement accuracy, the average measurement of the two systems for 100 samples was measured. The average WHR measurement was found to be 86.76 and $87.06 \mathrm{bpm}$ for the proposed system and BM system, respectively. Consequently, the WHR measurement accuracy was calculated based on Equation (4) [41] and found to be 99.65\%. The calculation of MAE and accuracy indicates that the proposed heart-rate measurement system measurements closely match the measurements of the BM system. 


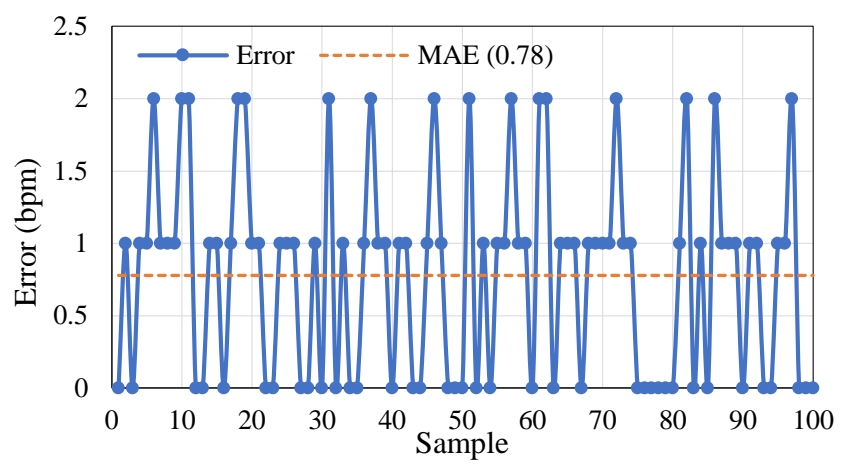

Figure 9. The measurement error of WHR absolute error.

\subsubsection{Bland-Altman Test}

Bland-Altman test can be used to show the agreement between the WHR and BM systems. The differences in a WHR measurement (i.e., errors) between the two systems are drawn on the $x$-axis, while the means of the two systems for each sample are drawn on the $y$ axis, as depicted in Figure 10. To create a Bland-Altman plot, several substantial parameters were computed, such as the standard deviation $(\sigma)$ and mean $(\mu)$ of the differences between the BM system and the WHR measurement system, the upper limit of agreements $(\mu+2 \sigma)$, lower limit of agreements $(\mu-2 \sigma)$, and the differences in WHR measurement between the two systems themselves.

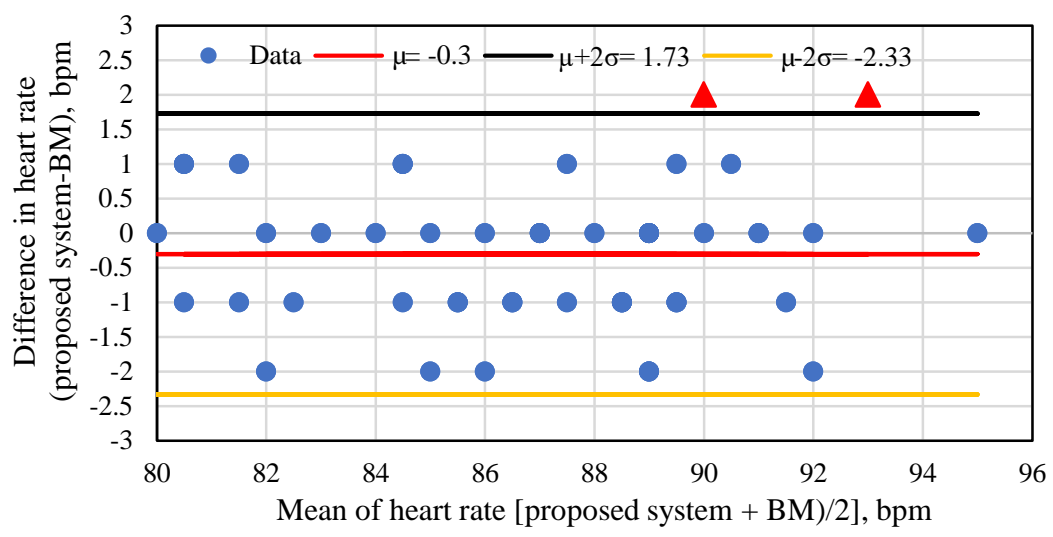

Figure 10. Bland-Altman plot for WHR measurements in beats per minute (bpm).

To investigate the similarity scores for the proposed system, four types of data are plotted in Figure 10: (i) the differences in WHR measurement between the two systems are plotted as blue circles (between the limits of agreement) and as red triangles (outside the limits of agreement); (ii) the mean of the difference (i.e., $\mu=-0.3$ ), plotted as a red line; (iii) the upper limit of the agreements (i.e., $\mu+2 \sigma=1.73$ ), as a black line; and (iv) the lower limit of agreements (i.e., $\mu-2 \sigma=-2.33$ ) as an orange line. In addition, the standard deviation $(\sigma)$ of the difference between the measurements of the two systems was calculated and found to be 1.015 . The variances within $\mu \pm 2 \sigma$ were found within $96 \%$ limits of the agreement between $(-2.33,1.73)$ at the average difference of the WHR measurements among the BM and proposed systems. The red triangles are outside the bounds (black and orange lines); the blue circle points are inside the bounds (black and orange lines). The width of parameters is $96 \%$ and the limit of the agreement is 4.06 in Figure 10. Thus, the Bland-Altman test reveals a relatively high agreement between the proposed WHR measurement system and the BM system. 


\subsubsection{Histogram Test}

A histogram is the most common method for graphical feature representation in terms of frequency distribution [45]. In this paper, the histogram includes neighboring bars or boxes; the $x$-axis denotes the bases of the bars representing the WHR measurements; the $y$-axis denotes the peaks of the bars representing the class counts or frequencies. Lower bars have lower scores, while higher bars suggest higher scores in a class. It is essential to detect whether the data of the implemented WHR measurement matches that of the BM system. The histogram plot in Figure 11 shows that the classes for heart rate are distributed between 80.4 and 97.03, with a minimum frequency of 2 and 4 in the classes. The histogram of the heart rate measurements shown in Figure 11 exhibited peak scores of 33 and 27 in the $91 \mathrm{bpm}$ classes for the BM system and proposed WHR measurement system, respectively. Further, the histogram of the WHR statistics shows peak scores of 28 and 22 in the $88.7 \mathrm{bpm}$ classes for the suggested WHR measurement system and BM device, respectively. These outcomes prove that the data obtained using the suggested WHR measurement system is similar to that obtained by the BM device.

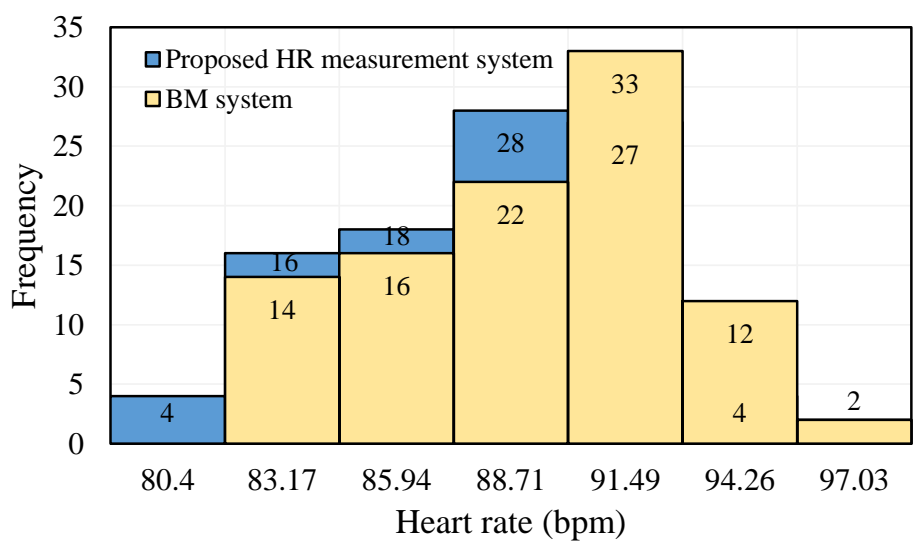

Figure 11. Histogram of the WHR data and BM device.

\section{Comparison with Previous Work}

The performance metrics of the proposed MRC-WPT-based WHR monitoring system can be compared with those found in previous studies. Several researchers have published papers on the different applications of WPT, and can be considered for comparison with the proposed system (Tables 4 and 5). Table 4 compares previous work in terms of Transfer efficiency, while Table 5 focuses on the power transfer conveyed to the resistive load. The proposed system outperforms the systems reported in these previous studies in terms of both efficiency and power transfer at different distances.

Table 4. Comparison of the current work with previous studies in terms of transfer efficiencies.

\begin{tabular}{ccccc}
\hline \multirow{2}{*}{ Ref./Year } & \multicolumn{4}{c}{ Transfer Efficiency (\%) at Different Distances } \\
\cline { 2 - 5 } & $\mathbf{3} \mathbf{~ c m}$ & $\mathbf{4} \mathbf{~ c m}$ & $\mathbf{5} \mathbf{~ c m}$ & $\mathbf{6} \mathbf{~ c m}$ \\
\hline$[27] / 2019$ & 35.58 & 17.5 & 7.8 & 6.3 \\
{$[46] / 2018$} & 42.56 & 40.33 & 40.26 & 40.11 \\
{$[47] / 2019$} & 57 & 56.9 & 56.5 & 56 \\
{$[48] / 2019$} & 33.24 & 20 & 10.4 & $\mathrm{~N} / \mathrm{A}$ \\
This work & $\mathbf{9 2 . 2}$ & $\mathbf{8 6 . 2 1}$ & $\mathbf{8 3 . 0 4}$ & $\mathbf{5 7 . 2 7}$ \\
\hline
\end{tabular}


Table 5. Comparison of the current work with previous studies in terms of transfer power.

\begin{tabular}{ccccc}
\hline \multirow{2}{*}{ Ref./Year } & \multicolumn{4}{c}{ Transfer Power (watt) at Different Distances } \\
\cline { 2 - 5 } & $\mathbf{3} \mathbf{~ c m}$ & $\mathbf{4} \mathbf{~ c m}$ & $\mathbf{6} \mathbf{~ c m}$ & $\mathbf{1 0} \mathbf{~ c m}$ \\
\hline$[27] / 2019$ & 8.54 & 4.2 & 1.5 & 1.05 \\
{$[46] / 2018$} & 2.55 & 2.42 & 2.41 & 1.39 \\
{$[49] / 2019$} & 11.4 & 11 & 10.6 & 9.4 \\
This work & $\mathbf{2 9 . 6}$ & $\mathbf{2 3 . 4 5}$ & $\mathbf{7 . 3 7 2}$ & $\mathbf{1 . 2}$ \\
\hline
\end{tabular}

Figure 12 compares the WHR measurement accuracy for the proposed system with those found in several previous articles [19-25,50-55] to confirm the performance of the suggested system. It can be seen that the proposed system accuracy was superior to that of other related works. However, the results in [50] were similar to the results in our current work.



Figure 12. Comparison of the current work with previous studies in terms of measurement accuracy.

\section{Conclusions}

This paper presented an MRC-WPT-based WHR monitoring system to solve the power supply problem of the heart rate measurement unit, which includes an Arduino Nano microcontroller, heart rate sensor, and nRF24L01 wireless communication module. The experimental results indicated that the proposed system can provide adequate power to operate the WHR measurement unit at an air gap of 1-15 cm between the receiver and transmitter coils. The power transfer and power Transfer efficiency were investigated and compared with those in earlier articles to verify the power achievement, where it was found that these two parameters outperformed values in previous articles. Moreover, the performance metrics of heart rate measurement were validated relative to a benchmark system through statistical analyses using error tests, the MAE test, the accuracy test, the Bland-Altman test, and the histogram test. In addition, the measurement accuracy was compared to that found in previous studies. The measurement accuracy was found to be $99.65 \%$, which was superior to that in most other studies. The results of this research are superior to those in previous articles regarding measurement accuracy, power transfer, and power Transfer efficiency. In future work, two or more low-power sensors should be embedded in one system to measure other biomedical parameters, such as temperature sensors, motion sensors, physical activity-based accelerometer sensors, ECG sensors, EMG sensors, and $\mathrm{SpO} 2$ sensors. In addition, increasing the output power will be studied to allow using the power system for supplying medium power consumption sensors. Moreover, future work may consider the adoption of misalignment conditions between transmitter and receiver coils such as the transverse, longitudinal, or angular offset of the coils where the transfer power and efficiency can be investigated for different conditions. 
Author Contributions: Conceptualization, M.F.M. and S.K.G.; methodology, M.F.M.; software, S.K.G.; validation, S.L.M., A.A.-N. and J.C.; formal analysis, M.F.M. and S.K.G.; investigation, A.A.-N.; resources, S.L.M.; data curation, M.F.M.; writing-original draft preparation, M.F.M. and S.K.G.; writing-review and editing, A.A.-N. and J.C.; visualization, S.K.G.; supervision, S.L.M.; project administration, J.C.; funding, A.A.-N. and J.C. All authors have read and agreed to the published version of the manuscript.

Funding: This research received no external funding.

Data Availability Statement: Not applicable.

Acknowledgments: The authors would like to thank the staff in the Department of Medical Instrumentation Techniques Engineering, Electrical Engineering Technical College, Middle Technical University-Baghdad, Iraq for their support to conduct this work.

Conflicts of Interest: The authors declare no conflict of interest.

\section{References}

1. Mahmood, M.F.; Mohammed, S.L.; Gharghan, S.K. Free Battery-based Energy Harvesting Techniques for Medical Devices. IOP Conf. Ser. Mater. Sci. Eng. 2020, 745, 012094. [CrossRef]

2. Mahmood, M.F.; Mohammed, S.L.; Gharghan, S.K. Ultrasound Sensor-Based Wireless Power Transfer for Low-Power Medical Devices. J. Low Power Electron. Appl. 2019, 9, 20. [CrossRef]

3. Curry, E.J.; Ke, K.; Chorsi, M.T.; Wrobel, K.S.; Miller, A.N.; Patel, A.; Kim, I.; Feng, J.; Yue, L.; Wu, Q. Biodegradable piezoelectric force sensor. Proc. Natl. Acad. Sci. USA 2018, 115, 909-914. [CrossRef] [PubMed]

4. Monti, G.; Arcuti, P.; Tarricone, L. Resonant Inductive Link for Remote Powering of Pacemakers. IEEE Trans. Microw. Theory Tech. 2015, 63, 3814-3822. [CrossRef]

5. Moore, J.; Castellanos, S.; Xu, S.; Wood, B.; Ren, H.; Tse, Z.T.H. Applications of wireless power transfer in medicine: State-of-the-art reviews. Ann. Biomed. Eng. 2019, 47, 22-38. [CrossRef] [PubMed]

6. Mahmood, M.F.; Mohammed, S.L.; Gharghan, S.K. Energy Harvesting-Based Vibration Sensor for Medical Electromyography Device. Int. J. Electr. Electron. Eng. Telecommun. 2020, 9, 364-372.

7. Thangasamy, V.; Kamsani, N.A.; Thiruchelvam, V.; Hamidon, M.N.; Hashim, S.J.; Bukhori, M.F.; Yusoff, Z. Wireless power transfer with on-chip inductor and class-E power amplifier for implant medical device applications. In Proceedings of the IEEE Student Conference on Research and Development (SCOReD), Kuala Lumpur, Malaysia, 13-14 December 2015; pp. $422-426$.

8. Ramachandran, H.; Bindu, G. Wireless powering of utility equipments in a smart home using magnetic resonance. In Proceedings of the IEEE Recent Advances in Intelligent Computational Systems (RAICS), Trivandrum, India, 19-21 December 2013; pp. 221-226.

9. Na, K.; Jang, H.; Ma, H.; Bien, F. Tracking optimal efficiency of magnetic resonance wireless power transfer system for biomedical capsule endoscopy. IEEE Trans. Microw. Theory Tech. 2014, 63, 295-304. [CrossRef]

10. Agarwal, K.; Jegadeesan, R.; Guo, Y.-X.; Thakor, N.V. Wireless power transfer strategies for implantable bioelectronics. IEEE Rev. Biomed. Eng. 2017, 10, 136-161. [CrossRef]

11. Li, X.; Tsui, C.-Y.; Ki, W.-H. A $13.56 \mathrm{MHz}$ wireless power transfer system with reconfigurable resonant regulating rectifier and wireless power control for implantable medical devices. IEEE J. Solid-State Circuits 2015, 50, 978-989. [CrossRef]

12. Zhao, J.; Ghannam, R.; Yuan, M.; Tam, H.; Imran, M.; Heidari, H. Design, test and optimization of inductive coupled coils for implantable biomedical devices. J. Low Power Electron. 2019, 15, 76-86. [CrossRef]

13. Gati, E.; Kokosis, S.; Patsourakis, N.; Manias, S. Comparison of Series Compensation Topologies for Inductive Chargers of Biomedical Implantable Devices. Electronics 2020, 9, 8. [CrossRef]

14. Herbert, R.; Yeo, W. Stretchable, Implantable Nanomembrane Biosensor for Wireless, Real-Time Monitoring of Hemodynamics. In Proceedings of the IEEE 69th Electronic Components and Technology Conference (ECTC), Las Vegas, NV, USA, 28-31 May 2019; pp. 1233-1239.

15. Kim, J.; Kim, H.; Kim, D.; Park, H.-J.; Ban, K.; Ahn, S.; Park, S.-M. A Wireless Power Transfer Based Implantable ECG Monitoring Device. Energies 2020, 13, 905. [CrossRef]

16. Jiang, Q.; Qin, Y.; Zhao, Y.; Xu, C.-Z.; Wang, X. A receiver position estimation scheme in wireless power transfer system. In Proceedings of the IEEE International Conference on Consumer Electronics (ICCE), Las Vegas, NV, USA, 12-14 January 2018; pp. 1-4.

17. Mohamadi, T. Working frequency in wireless power transfer for implantable biomedical sensors. In Proceedings of the 2011 International Conference on Electrical Engineering and Informatics, Bandung, Indonesia, 17-19 July 2011; pp. 1-5.

18. Zhang, F.; Hackworth, S.A.; Liu, X.; Chen, H.; Sclabassi, R.J.; Sun, M. Wireless energy transfer platform for medical sensors and implantable devices. In Proceedings of the 2009 Annual International Conference of the IEEE Engineering in Medicine and Biology Society, Minneapolis, MN, USA, 3-6 September 2009; pp. 1045-1048.

19. Campi, T.; Cruciani, S.; Feliziani, M.; Hirata, A. Wireless power transfer system applied to an active implantable medical device. In Proceedings of the 2014 IEEE Wireless Power Transfer Conference, Jeju, Korea, 8-9 May 2014; pp. $134-137$. 
20. Gore, V.B.; Gawali, D.H. Wireless power transfer technology for medical applications. In Proceedings of the 2016 Conference on Advances in Signal Processing (CASP), Pune, India, 9-11 June 2016; pp. 455-460.

21. Hussain, M.A.; Gharghan, S.K.; Qasim Hamood, H. Design and Implementation of Wireless Low-Power Transfer for Medical Implant Devices. IOP Conf. Ser. Mater. Sci. Eng. 2020, 745, 012087. [CrossRef]

22. Zhang, Z.; Chau, K.; Liu, C.; Li, F.; Ching, T. Quantitative analysis of mutual inductance for optimal wireless power transfer via magnetic resonant coupling. IEEE Trans. Magn. 2014, 50, 1-4. [CrossRef]

23. Zhang, Y.; Lu, T.; Zhao, Z.; He, F.; Chen, K.; Yuan, L. Employing load coils for multiple loads of resonant wireless power transfer. IEEE Trans. Power Electron. 2015, 30, 6174-6181. [CrossRef]

24. Campi, T.; Cruciani, S.; De Santis, V.; Maradei, F.; Feliziani, M. Near field wireless powering of deep medical implants. Energies 2019, 12, 2720. [CrossRef]

25. Zakaria, N.; Jusoh, M.; Ghazali, N.; Yasin, M.; Sabapathy, T.; Osman, M.; Ahmad, N.; Zakaria, M. A Computational Study on the Magnetic Resonance Coupling Technique for Wireless Power Transfer. In Proceedings of the MATEC Web of Conferences, Penang, Malaysia, 9-10 October 2017; p. 01025.

26. Luo, Z.; Wei, X. Mutual Inductance Analysis of Planar Coils with Misalignment for Wireless Power Transfer Systems in Electric Vehicle. In Proceedings of the 2016 IEEE Vehicle Power and Propulsion Conference (VPPC), Hangzhou, China, 17-20 October 2016; pp. 1-6.

27. Jawad, A.M.; Jawad, H.M.; Nordin, R.; Gharghan, S.K.; Abdullah, N.F.; Abu-Alshaeer, M.J. Wireless power transfer with magnetic resonator coupling and sleep/active strategy for a drone charging station in smart agriculture. IEEE Access 2019, 7, 139839-139851. [CrossRef]

28. Pantic, Z.; Lukic, S.M. Framework and topology for active tuning of parallel compensated receivers in power transfer systems. IEEE Trans. Power Electron. 2012, 27, 4503-4513. [CrossRef]

29. Mahmood, M.F.; Mohammed, S.L.; Gharghan, S.K.; Al-Naji, A.; Chahl, J. Hybrid Coils-Based Wireless Power Transfer for Intelligent Sensors. Sensors 2020, 20, 2549. [CrossRef] [PubMed]

30. Pérez-Nicoli, P. Inductive Links for Wireless Power Transfer: Fundamental Concepts for Designing High-Efficiency Wireless Power Transfer Links; Springer: Cham, Switzerland, 2021; p. 18.

31. Campi, T.; Cruciani, S.; De Santis, V.; Feliziani, M. EMF safety and thermal aspects in a pacemaker equipped with a wireless power transfer system working at low frequency. IEEE Trans. Microw. Theory Tech. 2016, 64, 375-382. [CrossRef]

32. Technical Specifications of Schottky Barrier Rectifier. Available online: http://www.datasheetcatalog.com/datasheets_pdf/S/R/ 2/6/SR260.shtml (accessed on 30 September 2020).

33. Furter, J.S.; Hauser, P.C. Interactive control of purpose built analytical instruments with Forth on microcontrollers-A tutorial. Anal. Chim. Acta 2019, 1058, 18-28. [CrossRef]

34. Gharghan, S.K.; Nordin, R.; Ismail, M. Energy Efficiency of Ultra-Low-Power Bicycle Wireless Sensor Networks Based on a Combination of Power Reduction Techniques. J. Sens. 2016, 2016, 7314207. [CrossRef]

35. Gharghan, S.K.; Nordin, R.; Ismail, M. An Ultra-Low Power Wireless Sensor Network for Bicycle Torque Performance Measurements. Sensors 2015, 15, 11741-11768. [CrossRef]

36. Thomas, S.S.; Saraswat, A.; Shashwat, A.; Bharti, V. Sensing heart beat and body temperature digitally using Arduino. In Proceedings of the 2016 International Conference on Signal Processing, Communication, Power and Embedded System (SCOPES), Paralakhemundi, India, 3-5 October 2016; pp. 1721-1724.

37. Valliappan, S.; Mohan, B.P.R.; Kumar, S.R. Design of low-cost, wearable remote health monitoring and alert system for elderly heart patients. In Proceedings of the 2017 International Conference on IoT and Application (ICIOT), Nagapattinam, India, 19-20 May 2017; pp. 1-7.

38. Zhao, L.; Thrimawithana, D.J.; Madawala, U.K. Hybrid bidirectional wireless EV charging system tolerant to pad misalignment. IEEE Trans. Ind. Electron. 2017, 64, 7079-7086. [CrossRef]

39. Parallax Data Acquisition Tool (PLX-DAQ) Software. Available online: https://www.parallax.com/package/plx-daq/ (accessed on 17 September 2021).

40. Gharghan, S.K.; Nordin, R.; Ismail, M. Statistical validation of performance of ZigBee-based wireless sensor network for track cycling. In Proceedings of the International Conference on Smart Sensors and Application (ICSSA), Kuala Lumpur, Malaysia, 26-28 May 2015; pp. 44-49.

41. Gharghan, S.K.; Mohammed, S.L.; Al-Naji, A.; Abu-AlShaeer, M.J.; Jawad, H.M.; Jawad, A.M.; Chahl, J. Accurate fall detection and localization for elderly people based on neural network and energy-efficient wireless sensor network. Energies 2018, 11, 2866. [CrossRef]

42. Arduino Nano. Available online: https://store.arduino.cc/usa/arduino-nano (accessed on 12 September 2020).

43. He, J.; Zhao, H. A new smart safety navigation system for cycling based on audio technology. Saf. Sci. 2020, 124, 104583. [CrossRef]

44. Kamel Gharghan, S.; Saad Fakhrulddin, S.; Al-Naji, A.; Chahl, J. Energy-Efficient Elderly Fall Detection System Based on Power Reduction and Wireless Power Transfer. Sensors 2019, 19, 4452. [CrossRef] [PubMed]

45. Gharghan, S.K.; Nordina, R.; Ismaila, M. Development and Validation of a Track Bicycle Instrument for Torque Measurement Using the Zigbee Wireless Sensor Network. Int. J. Smart Sens. Intell. Syst. 2017, 10, 124-145. 
46. Jawad, A.M.; Nordin, R.; Gharghan, S.K.; Jawad, H.M.; Ismail, M.; Abu-AlShaeer, M.J. Single-tube and multi-turn coil near-field wireless power transfer for low-power home appliances. Energies 2018, 11, 1969. [CrossRef]

47. Yang, C.; He, Y.; Qu, H.; Wu, J.; Hou, Z.; Lin, Z.; Cai, C. Analysis, design and implement of asymmetric coupled wireless power transfer systems for unmanned aerial vehicles. AIP Adv. 2019, 9, 025206. [CrossRef]

48. Ben Fadhel, Y.; Ktata, S.; Sedraoui, K.; Rahmani, S.; Al-Haddad, K. A modified wireless power transfer system for medical implants. Energies 2019, 12, 1890. [CrossRef]

49. Hussain, M.A.; Gharghan, S.K.; Hamood, H.Q. Single Tube Copper Coil via Strong Coupling Wireless Power Transfer for Low Power Devices. J. Adv. Res. Dyn. Control Syst. 2019, 11, 1818-1827.

50. Fakhri, A.B.; Gharghan, S.K.; Mohammed, S.L. Statistical Validation of Patient Vital Signs Based on Energy-efficient wireless sensor network monitoring system. ARPN J. Eng. Appl. Sci. 2018, 13, 8258-8270.

51. Roh, T.; Hong, S.; Yoo, H.-J. Wearable depression monitoring system with heart-rate variability. In Proceedings of the 36th Annual International Conference of the IEEE Engineering in Medicine and Biology Society, Chicago, IL, USA, 26-30 August 2014; pp. 562-565.

52. Zhou, C.-C.; Tu, C.-L.; Gao, Y.; Wang, F.-X.; Gong, H.-W.; Lian, P.; He, C.; Ye, X.-S. A low-power, wireless, wrist-worn device for long time heart rate monitoring and fall detection. In Proceedings of the International Conference on Orange Technologies, $\mathrm{Xi}^{\prime}$ an, China, 20-23 September 2014; pp. 33-36.

53. Ngu, A.; Wu, Y.; Zare, H.; Polican, A.; Yarbrough, B.; Yao, L. Fall Detection Using Smartwatch Sensor Data with Accessor Architecture. In Proceedings of the ICSH: International Conference on Smart Health, Hong Kong, China, 26-27 June 2017; pp. 81-93.

54. Di Rosa, M.; Hausdorff, J.M.; Stara, V.; Rossi, L.; Glynn, L.; Casey, M.; Burkard, S.; Cherubini, A. Concurrent validation of an index to estimate fall risk in community dwelling seniors through a wireless sensor insole system: A pilot study. Gait Posture 2017, 55, 6-11. [CrossRef]

55. He, J.; Bai, S.; Wang, X. An Unobtrusive Fall Detection and Alerting System Based on Kalman Filter and Bayes Network Classifier. Sensors 2017, 17, 1393. [CrossRef] [PubMed] 